\title{
The aryne phosphate reaction
}

\author{
Thomas M. Haas ${ }^{[a,+]}$ Stefan Wiesler, ${ }^{[a,+]}$ Tobias Dürr-Mayer, ${ }^{[a]}$ Alexander Ripp,,${ }^{[a, b]}$ Paraskevi Fouka, ${ }^{[a]}$ \\ Danye Qiu, ${ }^{[a]}$ Henning J. Jessen ${ }^{*[a, b]}$
}

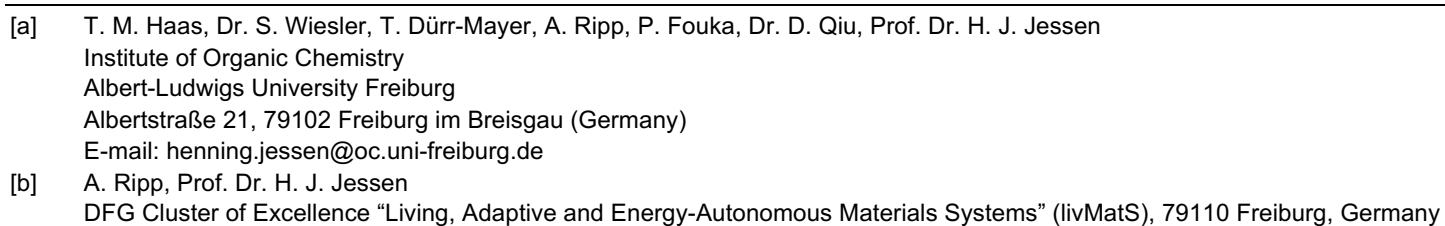

$\left[{ }^{+}\right]$These authors contributed equally to this work. $\left[{ }^{++}\right]$co-first authors

Supporting information for this article is given via a link at the end of the document

\begin{abstract}
Condensed phosphates are a critically important class of molecules in biochemistry, with a myriad of derived structures being known. Moreover, non-natural analogues are important for various applications, such as single molecule real time DNA sequencing. Often, such analogues contain more than three phosphate units in their oligophosphate chain. Consequently, investigations into phosphate reactivity enabling new ways of phosphate functionalization and oligophosphorylation are an essential endeavour in the field. Here, we scrutinize the potential of phosphates to act as arynophiles, paving the way for follow-up oligophosphorylation reactions. The aryne phosphate reaction is a powerful tool to - depending on the perspective (oligo)phosphorylate arenes or arylate (oligo-cyclo)phosphates. Based on Kobayashi-type o-silylaryltriflates, the aryne phosphate reaction enables rapid entry into a broad spectrum of arylated products, like monophosphates, diphosphates, phosphodiesters and polyphosphates. The synthetic potential of these new transformations is demonstrated by efficient syntheses of nucleotide analogues and an unprecedented one-flask octaphosphorylation.
\end{abstract}

\section{Introduction}

Arynes are "highly reactive, short-lived intermediates that can undergo a variety of chemical transformations." ${ }^{[1]}$ The simplest representative is 1,2-didehydrobenzene also known as benzyne (figure 1). The central structural motif of arynes is a bent triplebond that is part of a strained aromatic (poly)-cycle. ${ }^{[2,3]}$ This special bonding situation results in a low-energy LUMO, ${ }^{[4]}$ leading to significant electrophilic reactivity. ${ }^{[5]}$ Consequently, arynes are captured efficiently by arynophiles in diverse reactions, like nucleophilic additions, pericyclic reactions, insertions or multicomponent processes. ${ }^{[6]}$ Due to this broad applicability, aryne-based transformations are a powerful toolbox in chemical synthesis, underlined by their use in complex synthetic sequences towards natural products. ${ }^{[7,8]}$

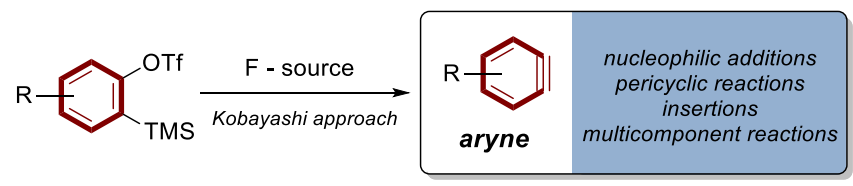

Figure 1. Aryne generation relying on Kobayashi's approach.
Because of their high reactivity and short lifetime, ${ }^{[9]}$ arynes are commonly generated in situ from stable precursors. Many strategies for aryne synthesis are available, ${ }^{[10]}$ and Kobayashiprecursors based on the o-silylaryltriflate functionality are particularly well developed. ${ }^{[11]}$ In this approach, fluoride ions induce aryne formation from bench-stable precursors in a temperature range in between $0{ }^{\circ} \mathrm{C}$ to $100^{\circ} \mathrm{C}{ }^{[8]}$

Arylated organophosphorous compounds are important building blocks in organic synthesis including various applications in medicinal chemistry, ${ }^{[12]}$ polymer science ${ }^{[13]}$ or as ligands in transition metal catalysis. ${ }^{[14,15]}$ Aryne-based methodologies have played a significant role in targeting such aryl-phosphorous compounds (scheme 1, A). ${ }^{[16,17]}$ In 2010, Jugé et al. presented a nucleophilic addition of phosphines to arynes leading to arylphosphonium salts (3). ${ }^{[18]} \mathrm{Biju}$ and He demonstrated later in 2014 and 2016 how this reactivity can be applied in multicomponent reactions with carbonyls or $\mathrm{CO}_{2} .^{[19,20]}$ Furthermore, Studer, Daugulis, and Miura developed aryne induced $\sigma-P^{\text {III }-X}$ bond insertions accessing ortho-stannylated, silylated or phosphinylated arylphosphines (6). ${ }^{[21-23]}$ Analogously, Alajarin and Gogoi presented $\pi-P^{\vee}=X$ bond insertions leading to ortho-aminated, thiolated or hydroxylated arylphosphonium products (8). ${ }^{[2,25]}$ Between 2013 and 2021 Mhaske, Chen and Willoughby applied phosphites, H-phosphonates and silylphosphates (11) to generate arylphosphonates (12). ${ }^{\text {26-28] }}$ Moreover, He presented a $\sigma-\mathrm{P}-\mathrm{O}-$-bond insertion of arynes into organophosphinic acids (9). ${ }^{[29]}$ In summary, the broad spectrum of aryne reactivity can be exploited with phosphorous functionalities towards diversely arylated products enabling C-P - bond formation. However, no exclusive aryne-based Oarylation has ever been described in the synthesis of organophosphorous compounds. More generally, "there are limited examples of $\mathrm{O}$-arylation transformations"[6] using arynes (scheme 1, B), overall restricted to few hydroxyl and carboxylgroup arylation protocols since 2004. ${ }^{\text {[30-32] }}$

Despite the many studies scrutinizing the reactivity of diverse arynophiles, the ability of phosphates to engage in reactions with arynes is absent from the literature. This is remarkable, as the urgent need to access probes for interrogation of phosphate functions, e.g. in analytical biochemistry, has driven innovative synthesis strategy development in the past years. ${ }^{[33-37]}$ 
A: Previous aryne chemistry of organophosphorous compounds

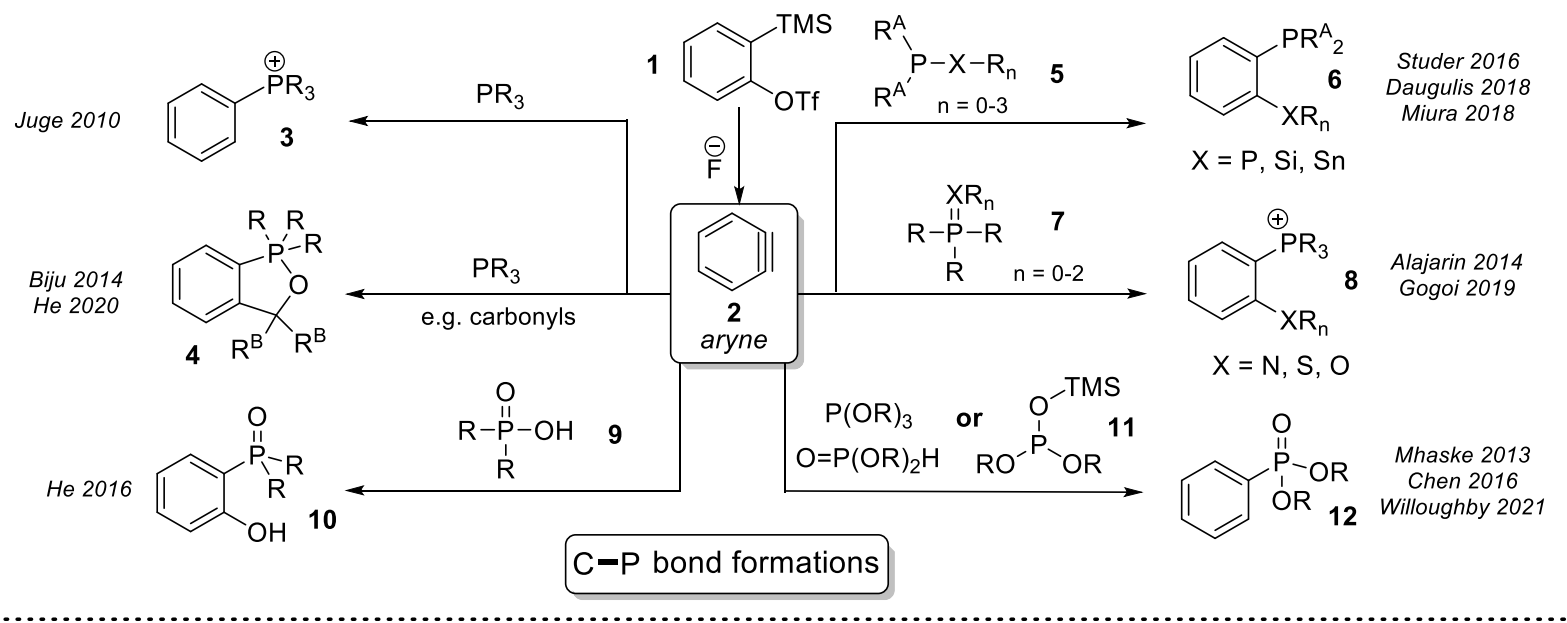

B: Previous 0-arylation chemistry with arynes<smiles>CS(=O)(=O)c1ccccc1O</smiles>

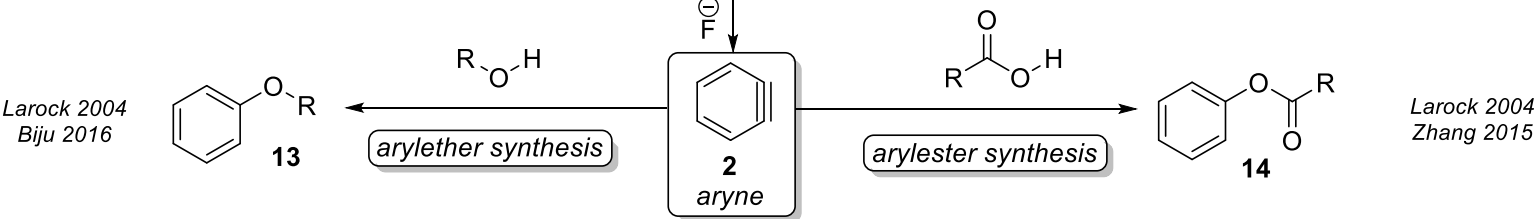

C: This work: The aryne phosphate reaction

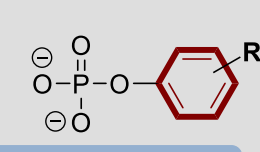

Arylmonophosphates
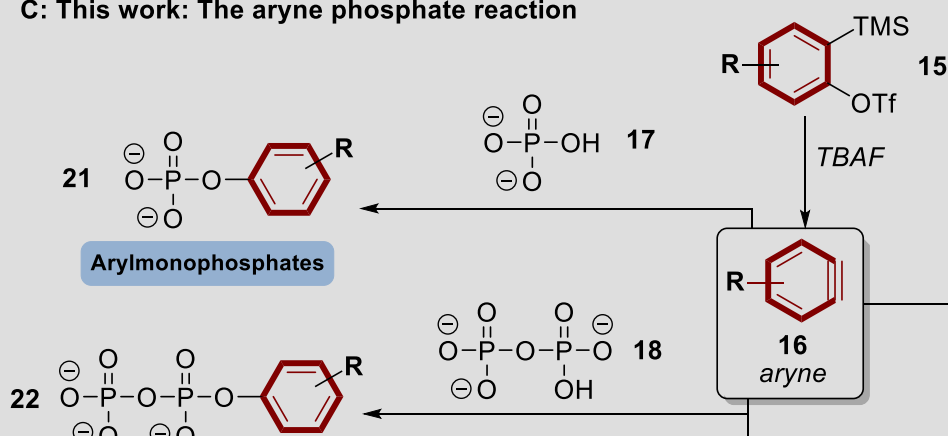

48 examples, up to $94 \%$ yield

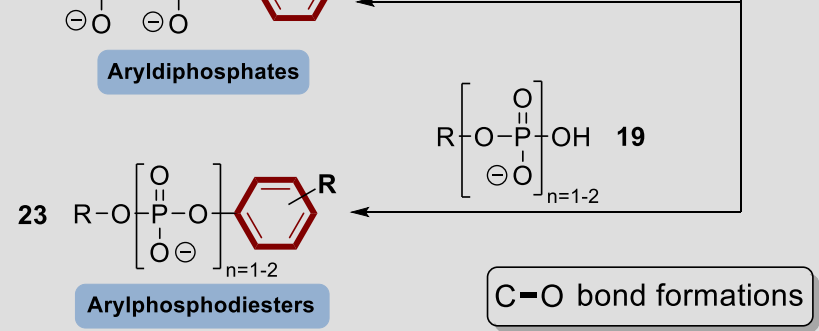

25

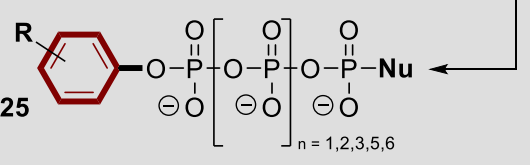

nucleophilic ring-opening

Scheme 1. A: Previously reported aryne chemistry of organophosphorous compounds based on o-silylphenyltriflates. B: Previously reported O-arylation chemistry of arynes based on o-silylaryltriflates. C: Synthetic concept of the aryne phosphate reaction including potentially accessible product groups.

Here, we present a detailed study of the phosphate aryne reaction. We demonstrate that phosphates, phosphate esters and anhydrides are arynophiles, which can be converted into $\mathrm{O}$ arylated phosphate derivatives. Starting from various substituted Kobayashi aryne precursors, we reacted the derived arynes with inorganic phosphate $\left(\mathrm{P}_{\mathrm{i}}, 17\right)$ and pyrophosphate $\left(\mathrm{PP}_{\mathrm{i}}, 18\right)$ towards arylated mono- (21) and diphosphates (22). In addition, phosphomonoesters (19) are selectively transformed into arylphosphodiesters (23). Notably, the method is applicable to cyclic condensed phosphates $(\mathbf{2 0}$, also known as metaphosphates), leading to storable arylated cyclophosphate species (24) of controllable ring-size, which have rarely been described. ${ }^{[38,39]}$ These species in turn are versatile oligophosphorylation reagents, linearizable by e.g. amine nucleophiles. We evaluate this concept up to an unprecedented one-flask octaphosphorylation reaction, but this appears not to be the limit. Overall, the scope of the aryne phosphate reaction 
is demonstrated in 48 examples covering a broad range of phosphates and aryne precursors.

\section{Results and Discussion}

Initial experiments were performed with Kobayashi's 2(trimethylsilyl)phenyltrifluoromethanesulfonate (1) and $\mathrm{PP}_{\mathrm{i}}$ (18) as a potential arynophile. An overview of the reaction optimization is shown in the SI (supporting table 1). In short, $\mathrm{MeCN}$ was identified as the optimal solvent, by enabling complete dissolution of the phosphates as tetrabutylammoniumsalts (TBA) while maintaining aryne reactivity. TBAF (1 $M$ in THF) was the ideal fluoride source as inorganic fluoride salts like $\mathrm{CsF}$ induced the precipitation of insoluble pyrophosphate metal salts. The rate and order of reagent addition turned out to play a critical role during reaction optimization. The highest yields were achieved, when TBAF was added slowly within $1 \mathrm{~h}$ via a syringepump to a solution of phosphate and aryne-precursor in MeCN. Notably, the reaction can be performed under ambient conditions without exclusion of moisture.

\section{Synthesis of (pyro)phosphomonoesters}

These optimized reaction conditions were applied in a first cluster of transformations: arynes derived from o-silylaryltriflates were reacted with $P_{i}(17)$ and $P P_{i}$ (18) leading to (pyro)phosphomonoesters (26, scheme 2, A). An excess of $P_{i}$ and $P P_{i}$ was applied in these transformations. In the case of $\mathrm{P}_{\mathrm{i}}, 60^{\circ} \mathrm{C}$ led to the highest yields, but $\mathrm{PP}$ i reactions required room temperature to avoid anhydride hydrolysis and over-arylation. The products (scheme 2, B) were purified chromatographically and usually isolated as TBA or TEA salts. Following this procedure, phenylphosphate 27 was synthesized in $88 \%$ yield. 2-Naphtylphosphate $\mathbf{2 8}$ was accessed from the corresponding naphtyne precursor in $65 \%$ yield and $3,4-$ dimethylphenylphosphate (29) was isolated in a yield of $92 \%$. Garg's indole 4,5-indolyne-precursor was transformed to indolylphosphate 30 in $41 \%$ yield, in a regioisomeric ratio of 96:4 (5-30:4-30). The observed regioisomeric preference towards C530 is in accordance with literature precedence and can be explained by Garg's aryne distortion model.[40]

Furthermore, we developed a new pyrene-derived aryneprecursor (SI-19) that was transformed successfully into the corresponding pyrenyl-phosphate $\mathbf{3 1}$ in $71 \%$ yield. The pyren-2yl regioisomer was preferentially formed in a ratio of 62:38. Presumably steric effects as well as aryne distortion synergistically direct the phosphate to pyrene's C2 position, ${ }^{[41]}$ further supported by an increase in selectivity for the bulkier PPi. We envision the reaction mechanism as a proton-coupled nucleophilic addition of phosphates (scheme 2, C) to arynes. Inter- and intramolecular proton transfer both seem plausible, as both water content in TBAF solutions and residual phosphate protons from the TBA salt, are suitable $\mathrm{H}^{+}$-sources. Consistently, the application of $\mathrm{MeCN}-\mathrm{d}_{3}$ as solvent did not lead to deuterium incorporation into the product (scheme $2 \mathrm{C}$ ).
While arguably arylmonophosphates are also accessible by alternative methods, ${ }^{[42,43]}$ the aryne phosphate reaction enables the straight-forward synthesis of otherwise difficult to access monoarylpyrophosphates. In fact, $\mathrm{PP}_{\mathrm{i}}$ proved to be a very efficient arynophile, leading to consistently high yields (scheme 2 , B). To ensure turnover at lowered reaction temperature, the $\mathrm{PP}_{\mathrm{i}}$ equivalents had to be raised compared to the $\mathrm{P}_{\mathrm{i}}$ case. Phenyldiphosphate 32, 3,4-dimethylphenyl-diphosphate 34 and pyrenyldiphosphate 36 were isolated in $>90 \%$ yields. 2-Naphtyldiphosphate 33 was obtained in $62 \%$ yield and indolyldiphosphate 35 was accessed in $42 \%$ yield.

Cluster I: Synthesis of (Pyro-)phosphomonoesters

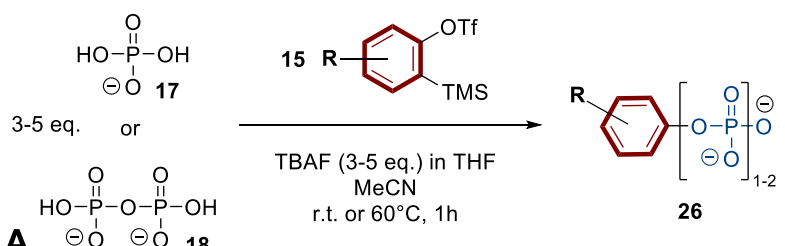

A $\odot$ O $\odot{ }^{\prime}$

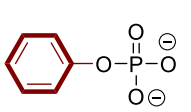

27: $88 \%$

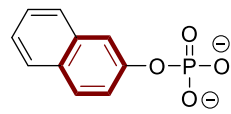

28: $65 \%$

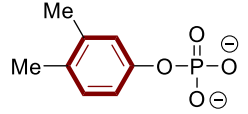

29: $92 \%$ $\longrightarrow$

30: $41 \%$

Indol-5-yl:Indol-4-yl 96:4

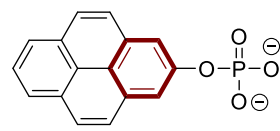

31: $71 \%$ para:meta $62: 38$<smiles>O=P([O-])(O)OP(=O)([O-])Oc1ccccc1</smiles>

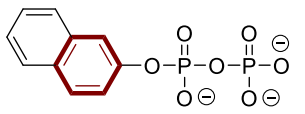

33: $62 \%$ 32: $91 \% \quad \mathrm{Me}$

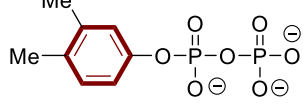

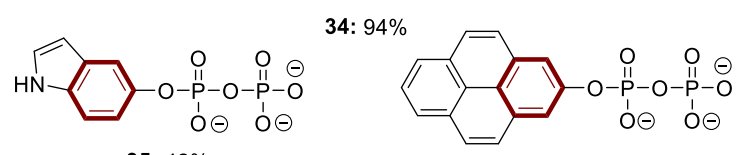

35: $42 \%$

Indol-5-yl:Indol-4-yl 96:4
36: $93 \%$

para:meta $82: 18$

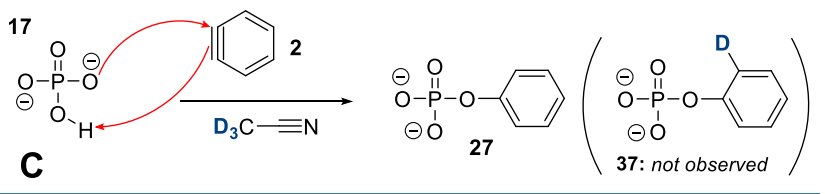

Scheme 2. Reaction cluster I. Synthesis of (pyro-)phosphomonoesters A: Synthetic concept. The reactions were performed on $300 \mu \mathrm{mol}$ scales. Monophosphate syntheses were performed at $60^{\circ} \mathrm{C}$, diphosphate syntheses at rt. $\mathrm{Pi}_{\mathrm{i}}$ and $\mathrm{PP}$ were used as TBA salts. The products were isolated as TEA and TBA salts or mixtures thereof. B: Substrate scope. C: Deuteration experiment. 
A

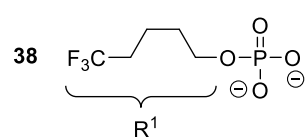

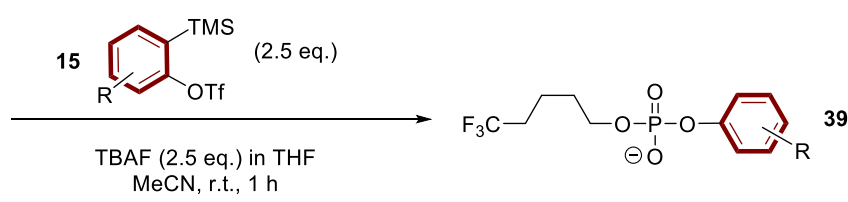

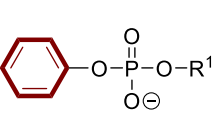

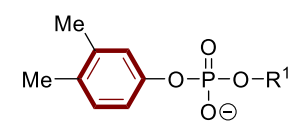

40: $51 \%$
41: $67 \%$

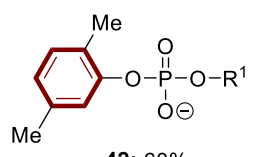

42: $60 \%$

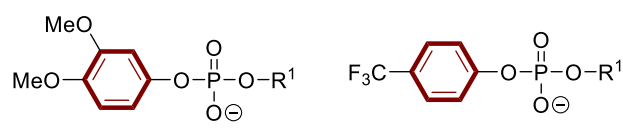

43: $57 \%$
$\overbrace{\substack{O-P_{1}-O-R^{1} \\ O}}^{O}$

45: $61 \%$<smiles>C#Cc1ccc(OP(=O)(O)O[Na])cc1</smiles>

\section{6: $64 \%$} (para:meta 63:37)

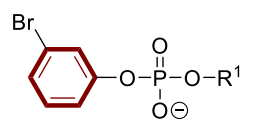

47: $57 \%$ (meta:ortho 88:12)
$\mathrm{Cl}$

48: $57 \%$ (para:meta 81:19)

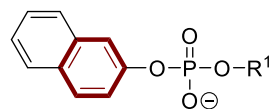

$\mathrm{O} \Theta$

49: $43 \%$

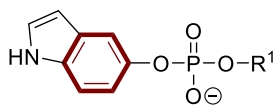

$\mathrm{O} \Theta$

B

50: $38 \%$

(Indol-5-yl:Indol-4-yl 92:8)

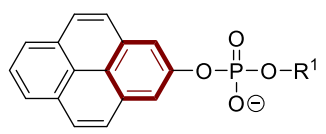

51: $58 \%$

(para:meta 78:22)

\section{Cluster II-B: Synthesis of Phosphodiesters: Phosphate - scope}

C

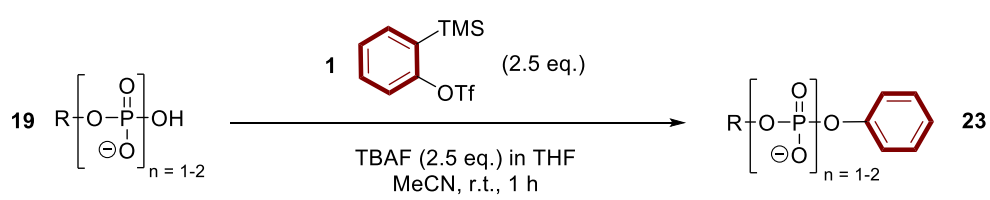

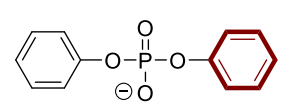

52: $55 \%$<smiles>C=C(C)CCOP(=O)(O)Oc1ccccc1</smiles>

56: $39 \%$

D

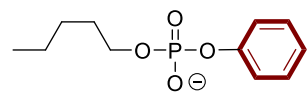

53: $56 \%$<smiles>C=CCC/C(C)=C/COP(=O)([O-])Oc1ccccc1</smiles>

57: $27 \%$

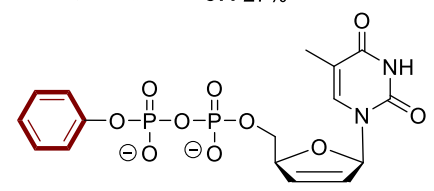

60: $33 \%$

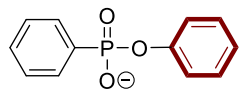

54: $46 \%$<smiles>O=P(O)(O)OCCCCCCO</smiles>

58: $15 \%$

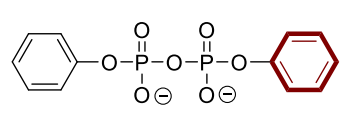

61: $47 \%$

Scheme 3. Reaction cluster II. Synthesis of arylphosphodiesters. Phosphates were introduced as TBA salts. The products are isolated as TEA or TBA salts. A Synthetic concept of aryne scope investigation. The reactions were performed on $300 \mu \mathrm{mol}$ scales and at concentrations of $200 \mathrm{mM}$. B: Aryne substrate scopes. C: Synthetic concept of phosphate monoester scope investigation. The reactions were performed on $150-500 \mu \mathrm{mol} s c a l e s$ and at concentrations of $200 \mathrm{mM}$. D: Phosphate monoester substrate scope.

\section{Synthesis of (pyro)phosphodiesters}

In a further cluster of experiments, we investigated the aryne phosphate reaction using phosphomonoesters as starting materials (scheme $3, \mathrm{~A}$ ). These transformations lead to phosphodiester products. 5,5,5-Trifluoropentylphosphate (38) was chosen as standard substrate in the aryne scope delineation (scheme $3, \mathrm{~A}+\mathrm{B}$ ), as the $\mathrm{CF}_{3}$-moiety can be used as an analytical handle for NMR-detection of products. 38 was reacted with 2.5 equivalents of $o$-silylaryltriflates and TBAF following the procedure described above. Only little overreaction to phosphate triesters was generally observed $(<10 \%)$ and these triesters usually decomposed during chromatographic purification. Under the applied conditions, $\mathbf{3 8}$ was successfully 
transformed into arylalkyldiester $\mathbf{4 0}$ in $51 \%$ yield. Dimethylphenyldiesters $\mathbf{4 1}$ and $\mathbf{4 2}$ were isolated in $67 \%$ and $60 \%$ yield, respectively. The reaction tolerates donor- and acceptor-substituted arynes delivering e.g. dimethoxyphenyland trifluoromethylphenyl diesters $\mathbf{4 3}$ and $\mathbf{4 4}$ yields of $57 \%$ and $50 \%$. In the case of unsymmetric 44 , the para-product was preferred in a ratio of $85: 15$. Sesamol-derived diester $\mathbf{4 5}$ was isolated in $61 \%$ yield. An alkynylated aryne precursor (SI-23) was developed and successfully applied generating 46 in $64 \%$ yield and a para:meta ratio of 84:16. Such clickable phosphordiester can be further functionalized by copper catalysed Huisgen-type cycloadditions. ${ }^{[44]} \mathrm{Br}$ - and $\mathrm{Cl}$-substituted diesters $(\mathbf{4 7}, \mathbf{4 8})$ were synthesized in comparable yields of $57 \%$, while the Br-substituent induced a higher regioselectivity. Furthermore, naphtyldiester 49 was isolated in $43 \%$ yield and indolyl-diester $\mathbf{5 0}$ was generated in a yield of $38 \%$ with a C5 positional preference of $85: 15$. Finally the pyryne was transformed to diester $\mathbf{5 1}$ in $58 \%$ yield and with a C2-preference of $78: 22$.

Subsequently, the phosphate scope was investigated using several organophosphates under the conditions described above (scheme 3, C+D). Diphenylphosphate 52 was synthesized in $55 \%$ yield. Similarly, pentylphenylphosphate $\mathbf{5 3}$ was isolated in $56 \%$ yield and alkynylated derivative $\mathbf{5 5}$ was obtained in $48 \%$. Notably, the reaction extends to phosphonates as demonstrated by the O-arylation of phenylphosphonate in $46 \%$ yield (54). In addition, terpenoide phosphates were successfully arylated leading to isoprenol derivative $\mathbf{5 6}$ in $\mathbf{3 9 \%}$ yield and the challenging geraniol-based allylphosphate 57 in $27 \%$ yield.

The chemoselectivity regarding hydroxy- and phosphate-groups - both O-nucleophiles - was studied using bifunctional 6hydroxy-hexylphosphate as a model substrate. Under the described reaction conditions, the phosphate group could however not be arylated selectively to give 58. Hydroxyarylation occurred to a comparable extent, leading to a modest yield of $15 \%$. This also explains that a highly selective phosphate arylation of unprotected nucleotides like adenosine monophosphate could not be achieved. In contrast, nucleotide analogue d4T-monophosphate was arylated successfully, generating 59 in $41 \%$ yield. Furthermore, also organodiphosphates were arylated, as demonstrated by d4Tdiphosphate arylation to derivative $\mathbf{6 0}$ in $33 \%$ yield. Finally, diphenylpyrophosphate (61) was isolated in $47 \%$ yield.

While overall good results were obtained in our cluster II studies, an obvious reduction in yields was observed as compared to cluster I. We now understand this limitation, as many reactions shown in scheme 3 also generated distinct byproducts, which can be explained mechanistically as outlined in scheme 4: In addition to pathway 1 leading to the desired products, byproduct formation according to pathway 2 was observed on the order of $25 \%$. In this case, the TBAF-solvent tetrahydrofuran (THF, 64) intercepts the aryne (2) under formation of cyclic oxonium-ion 65 Subsequently, the corresponding phosphate 62 attacks 65 in an $\mathrm{S}_{\mathrm{N} 2}$-process leading to isolable byproduct 66 . The noninnocence of THF towards arynes was already reported in $1970^{[45]}$ and is sometimes exploited synthetically in multicomponent approaches. ${ }^{[46]}$ Suppression of pathway 2, by changing TBAF-solvent from THF to MeCN (scheme 4, B), should therefore increase the yields significantly. We demonstrate this with pentylphosphate (67), where solvent exchange led to the substantially improved yield of $72 \%$ as compared to $56 \%$ when the medium contained THF. Therefore, it should be possible to increase the yields of the reactions summarized in scheme 3 at the expense of an additional solvent exchange procedure. Of note, the THF-derived byproducts were not observed for cluster I reactions (scheme 2), as in these cases $\mathrm{P}_{i}$ and $\mathrm{PP}_{\mathrm{i}}$ were applied in excess accompanied by reduced TBAF-equivalents.

\section{Suppression of byproduct formation}

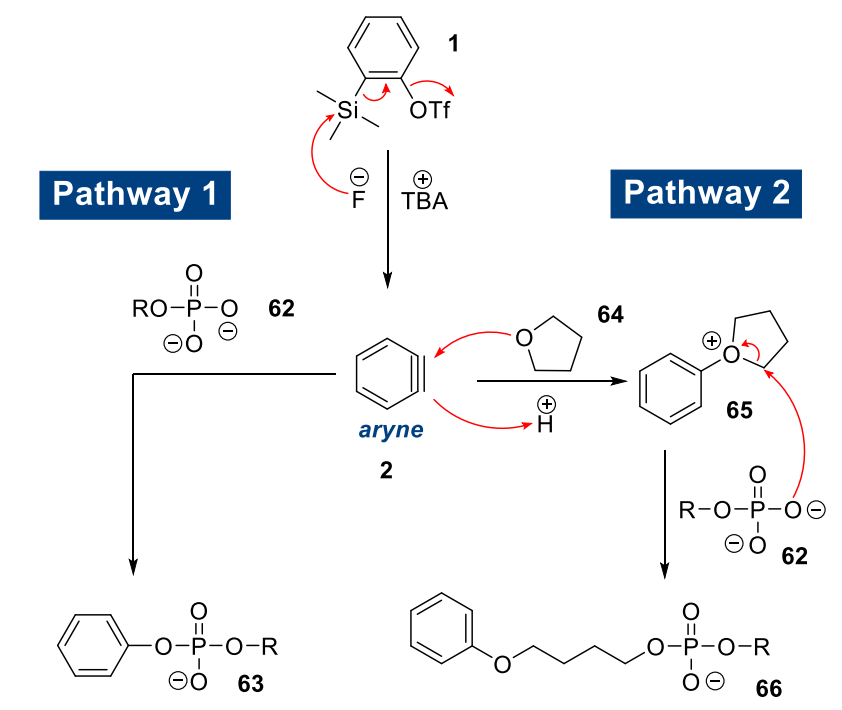

A desired product

byproduct (up to $25 \%$ )

Scheme 4. A: Depiction of product formation (pathway 1) and byproduct formation (pathway 2). B: Successful byproduct suppression by TBAF-solvent exchange increases the yield significantly.

\section{Synthesis of arylpolyphosphates}

In a third cluster of aryne phosphate reactions, the reactivity of arynes towards cyclophosphates was explored (scheme 5, A). Initial experiments with trimetaphosphate (68) using optimized conditions from scheme 2 proved the formation of arylcyclotriphosphate $\mathbf{7 3}$, but 4-5 eq. of aryne precursor were necessary to ensure complete turnover. Apart from that, the reaction conditions were kept constant. Notably, activation of cyclophosphates by arylation is not restricted to trimetaphosphate (68), but can also be applied to tetra- (69), penta- (70), hepta-, (71) and octametaphosphate (72). Monoarylcyclophosphates $\mathbf{7 3}$ and $\mathbf{7 4}$ could be isolated as oils after precipitation and stored as reagents for several weeks at $-20^{\circ} \mathrm{C}$ without substantial decomposition. Hence, $\mathbf{7 3}$ and $\mathbf{7 4}$ are storable tri- and tetraphosphorylation reagents. 


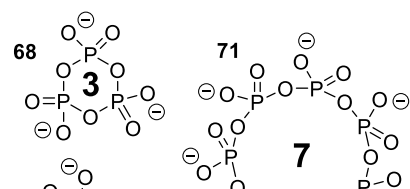

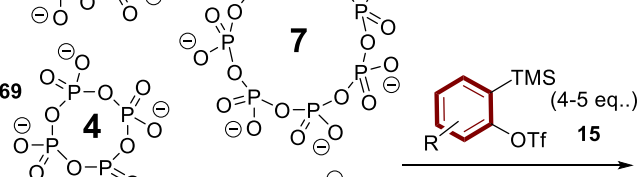

$O^{\prime \prime} O-P_{1}^{\prime}=0$
$O_{\ominus}$

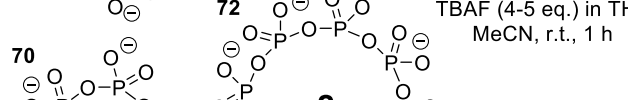

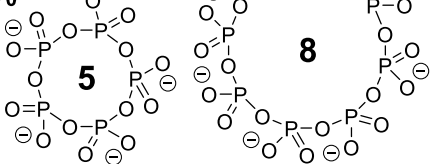

A

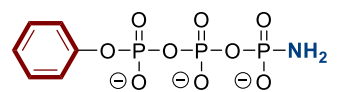

79: $67 \%$
Triphosphates

Tetraphosphates

Pentaphosphates

Heptaphosphates

Octaphosphates

(2:<smiles>C#CCNP(=O)(O)OP(=O)(O)OP(=O)(O)OP(=O)(O)OP(=O)(O)Oc1ccccc1</smiles>
pyren-2-yl : pyren-1-yl = 88:12

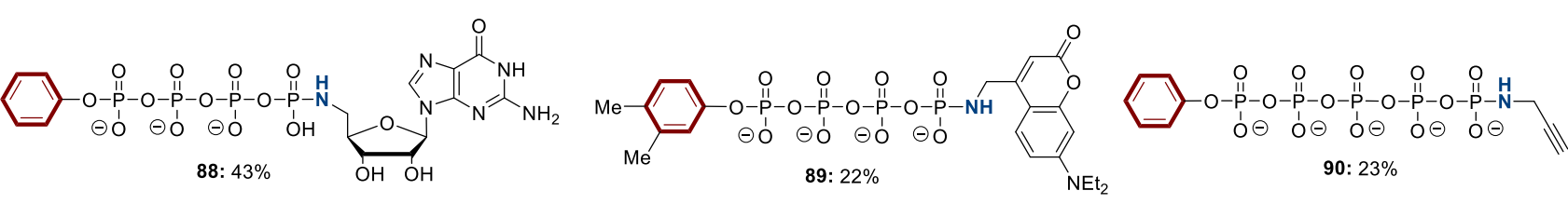

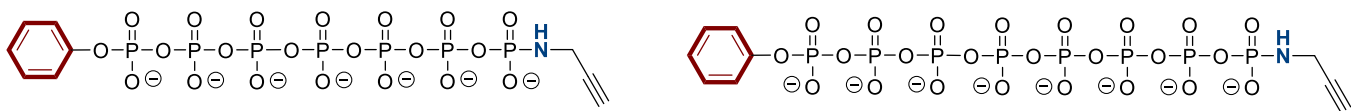

B

91: $37 \%$

92: $22 \%$

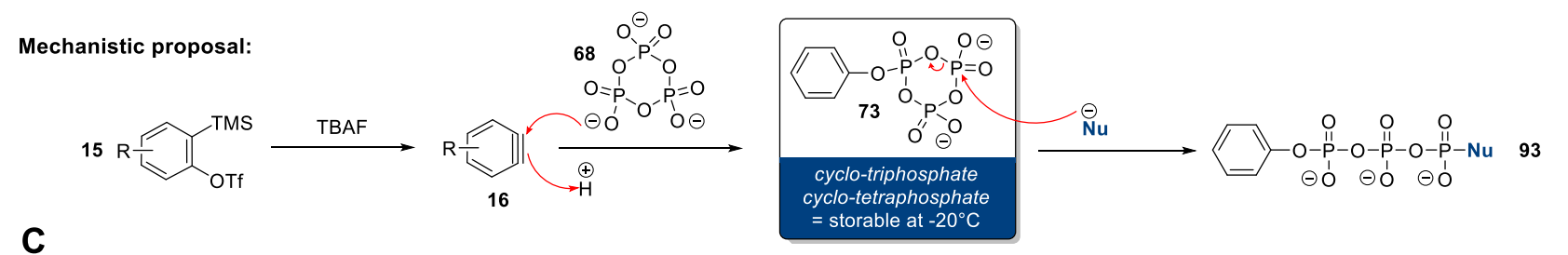

Scheme 5. A: Synthetic concept of reaction cluster III. Arylation of cyclophosphates followed by nucleophilic ring-opening. The reactions were performed on $100 \mu \mathrm{mol}$ scales and at concentrations of $70 \mathrm{mM}$. For $\mathrm{P}_{5}-\mathrm{P}_{8}$ syntheses, the amount of aryne precursor (4.0 eq.) and nucleophile (20 eq.) differed from $\mathrm{P}_{3}$ - $\mathrm{P}_{4}$ syntheses [aryne precursor (5.0 eq.), nucleophile (2.5 eq.)]. B: Substrate scope of arylpolyphosphate synthesis. C: Mechanistic proposal of nucleophilic ringopenings. 
All arylcyclophosphates 73-77 were subsequently linearized using $\mathrm{N}$-nucleophiles, based on an $\mathrm{S}_{\mathrm{N}}$ type mechanism (scheme $5, \mathrm{C}) .{ }^{[34]}$ The reaction sequence enabled the isolation of various arylated polyphosphate chains $\left(P_{3}-P_{8}\right.$, scheme $\left.5, B\right)$. Most polyphosphate products were purified by automated strong-ion exchange chromatography (SAX) and isolated as $\mathrm{Na}$ salts.

Using Kobayashi's o-silylphenyltriflate (1), trimetaphosphate (68) was smoothly transformed into phenyltrimetaphosphate (73), which was then ring-opened by several amine nucleophiles generating the terminally modified triphosphates 78-85. For example, propargylamidotriphosphate $\mathbf{7 8}$ and amidotriphosphate 79 were isolated in overall yields of $69 \%$ and $67 \%$ from trimetaphosphate. Ring-opening with anthracenylmethanamine led to triphosphate $\mathbf{8 0}$ in $46 \%$ yield. Furthermore, phenylcyclotriphosphate could be linearized with $5^{\prime}$ aminonucleosides, such as aminoadenosine SI-1 and aminoguanosine SI-2 leading to the nucleotide analogues $\mathbf{8 1}$ and 82 in $53 \%$ and $66 \%$ yield, respectively. Alternative aryne precursors were coupled analogously with trimetaphosphate, exemplified in naphtylated GTP analogue 83 , accessed in $49 \%$ yield. The ATP-derived pyrenyltriphosphate $\mathbf{8 4}$ was isolated in $41 \%$ yield. The higher steric demand of cyclotriphosphate compared to $P_{i}$ (scheme 2) is reflected in an increased regioisomeric C2-preference of 88:12. In addition, sesamylcyclotriphosphate was opened with amino-DEACM (SI26) towards the fluorescent triphosphate analog 85 in $59 \%$ yield. Likewise, tetrametaphosphate was efficiently arylated using osilylaryltriflate precursors. Propargylamidotetraphosphate $\mathbf{8 6}$ was synthesized in $41 \%$ yield. Ring-opening of phenyltetraphosphate with aminoguanosine generated G4Panalogue 88 in $43 \%$ yield. Furthermore, the synthesis of pyrenylated G4P (87) was achieved in $45 \%$ yield. The regioselectivity was similar to cyclotriphosphate example 84 . Fluorescent dimethylphenyltetraphosphate 89 was accessed via amino-DEACM in $22 \%$ yield.

Propargylamidopentaphosphate $\mathbf{9 0}$ was isolated in $23 \%$ yield from pentametaphosphate (70), by ring-opening of phenylcyclopenta-phosphate 75. Analogously, heptaphosphate 91 and octaphosphate 92 were isolated in yields of $37 \%$ and $22 \%$, respectively, from their corresponding metaphosphate precursors. In the cases of polyphosphates $\mathrm{P}>4$ the nucleophile equivalents were increased from 2.5 to 10 , to ensure smooth ring-opening.

\section{Conclusion}

In 2017 Garg stated about arynes that "their high reactivity has seemingly steered chemists away from using them to assemble [...] complex scaffolds. [But] arynes can and should be used strategically to enable the synthesis of complex molecules with motifs that have conventionally been viewed as challenging." ${ }^{[47]}$ In the present paper, we meet this suggestion by introducing the aryne phosphate reaction that accesses highly challenging arylated organophosphorous compounds with Kobayashi-type precursors. We demonstrate that inorganic phosphates, organophosphates and cyclophosphates are efficient arynophiles in O-arylation reactions. An extensive portfolio of products was presented, ranging from arylmonophosphates and -diphosphates to arylphosphodiesters and -polyphosphates. Yet, there are countless more combinations that can be envisioned to further expand the repertoire of the aryne phosphate reaction. The versatility of this novel transformation is further demonstrated by synthetically unlocking penta-, hepta- and octaphosphorylations in one-flask operations. Polyphosphorylation reagents introducing defined phosphate chains with more than four units in a single reaction have never been reported before. Consequently, the disclosed penta-, hepta- and octaphosphorylation procedures set new benchmarks in polyphosphorylation chemistry. It is apparent that longer polyP chains could potentially be accessible by either extending the ring-size or by using phosphate nucleophiles on arylated cyclophosphates. We are confident that the aryne phosphate reaction will stimulate tool design in nucleotide and polyP research and - more generally - enable us to venture into the largely uncharted and fascinating realm of condensed phosphates.

\section{Acknowledgements}

We thank Dr. Manfred Keller from MagRes of the University of Freiburg for a significant amount of time for NMR spectroscopy and Christoph Warth for HRMS measurements. This work was funded by the Deutsche Forschungsgemeinschaft (DFG, German Research Foundation) under Germany's Excellence Strategy-EXC-2193/1-390951807 via "Living, Adaptive and Energy-Autonomous Materials Systems" (livMatS). This project has received funding from the European Research Council (ERC) under the European Union's Horizon 2020 research and innovation program (grant agreement no. 864246, to H.J.J). We gratefully acknowledge financial support from the Studienstiftung des Deutschen Volkes, the Brigitte Schlieben-Lange Programm, and Cusanus-Werk.

Keywords: Aryne chemistry - Phosphorylation • oligophosphorylation $\cdot$ metaphosphates $\cdot$ polyphosphates

[1] J. T. S. Yeoman, S. E. Reisman, Nature 2012, 490, 179-180.

[2] H. H. Wenk, M. Winkler, W. Sander, Angew. Chem. Int. Ed. 2003, 42, 502-528.

[3] In this Paper the expression aryne covers ortho-arynes only.

[4] N. G. Rondan, L. N. Domelsmith, K. N. Houk, A. T. Bowne, R. H. Levin, Tetrahedron Lett. 1979, 35, 3237-3240.

[5] N. F. F. Nathel, L. A. Morrill, H. Mayr, N. K. Garg, J. Am. Chem. Soc. 2016, 138, 10402-10405.

[6] J. Shi, L. Li, Y. Li, Chem. Rev. 2021, 121, 3892-4044.

[7] C. M. Gampe, E. M. Carreira, Angew. Chem. Int. Ed. 2012, 51, 37663778.

[8] H. Takikawa, A. Nishii, T. Sakai, K. Suzuki, Chem. Soc. Rev. 2018, 47, 8030-8056.

[9] N. Pavliček, B. Schuler, S. Collazos, N. Moll, D. Pérez, E. Guitián, G. Meyer, D. Peña, L. Gross, Nature Chem. 2015, 7, 623-628.

[10] S. Yoshida, T. Hosoya, Chem. Lett. 2015, 44, 1450-1460.

[11] Y. Himeshima, T. Sonada, H. Kobayashi, Chem. Lett. 1983, 8, 12111214.

[12] M. Sawa, T. Kiyoi, K. Kurokawa, H. Kumihara, M. Yamamoto, T. Miyasaka, Y. Ito, R. Hirayama, T. Inoue, Y. Kirii, E. Nishiwaki, H. Ohomoto, Y. Maeda, E. Ishibushi, Y. Inoue, K. Yoshino, H. Kondo, J. Med. Chem. 2002, 45, 919-929.

[13] T. Baumgartner, R. Reau, Chem. Rev. 2006, 106, 4681-4727.

[14] E. I. Musina, A. S. Balueva, A. A. Karasik, Organophosphorous Chem. 2019, 48, 1-63. 
[15] V. V. Grushin, Chem. Rev. 2004, 104, 1629-1662.

[16] J. Chen, R. Fan, Z. Liu, J. Tan, Adv. Synth. Catal. 2021, 363, 657-667.

[17] K. Neog, P. Gogoi, Org. Biomol. Chem. 2020, 18, 9549-9561.

[18] E. Rémond, A. Tessier, F. R. Leroux, J. Bayardon, S. Jugé, Org. Lett. 2010, 12, 1568-1571.

[19] A. Bhunia, T. Roy, R. G. Gonnade, A. T. Biju, Org. Lett. 2014, 16, 51325135.

[20] P. Xie, S. Yang, Y. Guo, Z. Cai, B. Dai, L. He, J. Org. Chem. 2020, 85 8872-8880.

[21] Y. Li, S. Chakrabarty, C. Mück-Lichtenfeld, A. Studer, Angew. Chem. Int. Ed. 2016, 55, 802-806.

[22] M. Mesgar, J. Nguyen-Le, O. Daugulis, J. Am. Chem. Soc. 2018, 140, 13703-13710.

[23] Y. Okugawa, Y. hayashi, S. Kawauchi, K. Hirano, M. Miura, Org. Lett. 2018, 20, 3670-3673.

[24] K. Neog, D. Dutta, B. Das, P. Gogoi, Org. Biomol. Chem. 2019, 17, 6450-6460.

[25] C. Lopez-Leonardo, R. Raja, F. López-Ortiz, M. Ángel del ÁguilaSánchez, M. Alajarin, Eur. J. Org. Chem. 2014, 1084-1095.

[26] R. A. Dhokale, S. B. Mhaske, Org. Lett. 2013, 15, 2218-2221

[27] Q. Chen, X. Yan, C. Wen, J. Zeng, Y. Huang, X. Liu, K. Zhang, J. Org. Chem. 2016, 81, 9476-9482.

[28] B. M. Bembenek, M. M. S. Petersen, J. A. Lilly, A. L. Haugen, N. J. Jiter A. J. Johnson, E. E. Ripp, S. A. Winchell, A. N. Harvat, C. McNulty, S. A. Thein, A. M. Grieger, B. J. Lyle, G. L. Mraz, A. M. Stitgen, S. Foss, M. L. Schmid, J. D. Scanlon, P. H. Willoughby, J. Org. Chem. 2021, 86, 10724-10746.

[29] N. Qi, N. Zhang, S. R. Allu, J. Gao, J. Guo, Y. He, Org. Lett. 2016, 18 , 6204-6207.

[30] Z. Liu, R. C. Larock, Org. Lett. 2004, 6, 99-102.

[31] C. Wen, Q. Chen, Z. He, X. Yan, C. Zhang, Z. Du, K. Zhang, Tetrahedron Lett. 2015, 56, 5470-5473.

[32] M. Thangaraj, S. S. Bhojgude, M. V. Mane, A. T. Biju, Chem. Commun. 2016, 52, 1665-1668.

[33] S. Mohamady, S. D. Taylor, Org. Lett. 2013, 15, 2612-2615.

[34] S. M. Shepard, H. Kim, Q. X. Bang, N. Alhokbany, C. C. Cummins, J. Am. Chem. Soc. 2021, 143, 463-470.

[35] J. Singh, A. Ripp, T. M. Haas, D. Qiu, M. Keller, P. A. Wender, J. S. Siegel, K. K. Baldrige, H. J. Jessen, J. Am. Chem. Soc. 2019, 141 15013-15017.

[36] J. Singh, N. Steck, D. De, A. Hofer, A. Ripp, I. Captain, M. Keller, P. A. Wender, R. Bhandari, H. J. Jessen, Angew. Chem. Int. Ed. 2019, 58, 3928-3933.

[37] T. Dürr-Mayer, D. Qiu, V. B. Eisenbeis, N. Steck, M. Häner, A. Hofer, A. Mayer, J. S. Siegel, K. K. Baldridge, H. J. Jessen, Nat. Commun. 2021, 12, 5368.

[38] S. M. Shepard, I. W. Windsor, R. T. Raines, C. C. Cummins, J. Am. Chem. Soc. 2019, 141, 18400-18404

[39] S. M. Shepard, C. C. Cummins, J. Am. Chem. Soc. 2019, 141, 1852 1856.

[40] E. Picazo, K. N. Houk, N. K. Garg, Tetrahedron Lett. 2015, 56, 3511 3514 .

[41] S. M. Bronner, J. L. Mackey, K. N. Houk, N. K. Garg, J. Am. Chem. Soc 2012, 134, 13966-13969.

[42] L. J. Silverberg, J. L. Dillon, P. Vemishetti, Tetrahedron Lett. 1996, 37, 771-774.

[43] S. E. Bunn, C. T. Liu, Z.-L. Lu, A. A. Neverov, R. S. Brown, J. Am Chem. Soc. 2007, 129, 16238-16248.

[44] M. Breugst, H.-U. Reissig, Angew. Chem. Int. Ed. 2020, 59, 1229312307.

[45] E. Wolthuis, B. Bouma, J. Modderman, L. Sytsma, Tetrahedron Lett. 1970, 11, 407-408

[46] H. Yoshida, Y. Asatsu, Y. Mimura, Y. Ito, J. Ohshita, K. Takaki, Angew. Chem. Int. Ed. 2011, 50, 9676-9679.

[47] M. A. Corsello, J. Kim, N. K. Garg, Nat. Chem. 2017, 9, 944-949. 
